\title{
Modeling Heavy Metal Sorption Kinetics Using Fractional Calculus
}

\author{
V. C. Friesen, ${ }^{1}$ D. P. Leitoles, ${ }^{1}$ G. Gonçalves, ${ }^{2}$ E. K. Lenzi, ${ }^{3}$ and M. K. Lenzi ${ }^{1}$ \\ ${ }^{1}$ Departamento de Engenharia Química, Universidade Federal do Paraná, Caixa Postal 19011, 81531-980 Curitiba, PR, Brazil \\ ${ }^{2}$ Departamento de Engenharia Química, Universidade Tecnológica Federal do Paraná, 84016-210 Ponta Grossa, PR, Brazil \\ ${ }^{3}$ Departamento de Física, Universidade Estadual de Ponta Grossa, 84030-900 Ponta Grossa, PR, Brazil
}

Correspondence should be addressed to M. K. Lenzi; lenzi@ufpr.br

Received 9 December 2014; Accepted 11 March 2015

Academic Editor: Bo Yu

Copyright $(2015$ V. C. Friesen et al. This is an open access article distributed under the Creative Commons Attribution License, which permits unrestricted use, distribution, and reproduction in any medium, provided the original work is properly cited.

Heavy metals are commonly regarded as environmentally aggressive and hazardous to human health. Among the different metals, lead plays an important economic role due to its large use in the automotive industry, being an essential component of batteries. Different approaches have been reported in the literature aimed at lead removal, and among them a very successful one considers the use of water hyacinths for sorption-based operation. The modeling of the metal sorption kinetics is a fundamental step towards in-depth studies and proper separation equipment design and optimization. Fractional calculus represents a novel approach and a growing research field for process modeling, which is based on the successful use of derivatives of arbitrary order. This paper reports the modeling of the kinetics of lead sorption by water hyacinths (Eichhornia crassipes) using a fractional calculus. A general procedure on error analysis is also employed to prove the actual fractional nature of the proposed model by the use of parametric variance analysis, which was carried out using two different approaches (with the complete Hessian matrix and with a simplified Hessian matrix). The joint parameter confidence regions were generated, allowing to successfully show the fractional nature of the model and the sorption process.

\section{Introduction}

Heavy metals, particularly lead, are widely present in industrial applications such as in paints [1] and in batteries [2]. The presence of lead represents an important environmental issue due to its high toxicity $[3,4]$. Therefore, several techniques have been successfully proposed to remove lead and other heavy metals from wastewater streams allowing a safe discharge. The most common techniques are based on chemical precipitation [5], ion exchange [6], electrochemical methods [2], and sorption/biosorption [7]. Over the past decade, several applications have reported the use of water hyacinths $[7,8]$ aimed at lead removal, providing a self-sustainable approach.

An essential tool for in-depth process studies, design, optimization, and control of processes concerning lead removal is given by mathematical models. They play a very important role as actual experimental runs cannot necessarily be carried out because accurate predictions and deviations can be obtained from simulation of different operating conditions [9]. This turns out to be a very efficient and economic approach for process studies, if the model has been properly derived and tuned by adequate parameter estimation and error analysis tasks [10].

Modeling tasks in heavy metal sorption are usually splitted into two parts: (i) equilibrium/isotherm modeling and (ii) kinetics modeling. Equilibrium/isotherm modeling usually concerns the use of classical models based on Langmuir [11], Freundlich [12], and Redlich and Peterson [13], among others [14]. On the other hand, kinetics modeling usually employs either first and pseudo-first order models [15] or second and pseudo-second order models [16].

Fractional calculus represents an innovative approach and a very fast growing research field for process modeling, based on derivatives of arbitrary order [17]. The literature reports a broad range of applications ranging from molecular diffusion processes [18] to industrial systems engineering [19]. Regarding heavy metals sorption modeling with 
TABLE 1: Experimental data for the lead sorption.

\begin{tabular}{|c|c|c|c|c|c|c|c|c|}
\hline \multicolumn{9}{|c|}{ RUN01 $-y_{0}=10 \mathrm{mgPb} / \mathrm{L}$} \\
\hline$y(t) / y_{0}$ & 1 & 0.479 & 0.292 & 0.214 & 0.142 & 0.075 & 0.049 & 0.049 \\
\hline$t-[$ day $]$ & 0 & 0.125 & 0.25 & 0.375 & 0.5 & 1 & 2 & 3 \\
\hline \multicolumn{9}{|c|}{ RUN02 $-y_{0}=15 \mathrm{mgPb} / \mathrm{L}$} \\
\hline$y(t) / y_{0}$ & 1 & 0.519 & 0.389 & 0.217 & 0.161 & 0.088 & 0.041 & 0.041 \\
\hline$t-[$ day $]$ & 0 & 0.125 & 0.25 & 0.375 & 0.5 & 1 & 2 & 3 \\
\hline
\end{tabular}

the aid of fractional calculus, Gomes et al. [10] reported a novel isotherm to describe the lead sorption behavior and also provide an in-depth methodology to analyze the actual fractional feature of the estimated order of the fractional derivative. Concerning fractional kinetics modeling, some milestone reported works provide the fundamental knowledge of the theoretical fractional kinetics $[20,21]$, while others report the application of the fractional modeling approach to different heavy metal sorption systems [22-25].

This work reports the modeling of the kinetic behavior of experimental lead sorption data using Eichhornia crassipes as the sorption medium. In order to describe the experimental behavior, a fractional kinetics model is considered and a comparison to integer order kinetic model is also performed. Differently from previously reported manuscripts, a parametric analysis [10] is carried out and joint parameter confidence regions are generated, being successfully able to show that the fractional nature of the model is statistically valid.

\section{Experimental Data}

The experimental data used for the modeling purposes were reported by dos Santos and Lenzi [8], where further details regarding the experimental procedures can be obtained. Table 1 presents a summary of the experimental datasets used. In both sets, the concentration of the water hyacinth was the same and equal to $20 \mathrm{~g}$ of hyacinth per liter of solution. This amount of hyacinth was submitted to two different initial concentrations of lead. In experimental run RUN01, this concentration was equal to $10 \mathrm{mg}$ of lead $(\mathrm{Pb}) / \mathrm{L}$, while in experimental run RUN02, the initial concentration was equal to $15 \mathrm{mg}$ of lead $(\mathrm{Pb}) / \mathrm{L}$. Prior to the parameter estimation tasks, the data were normalized by dividing all values by the initial condition (concentration). Finally it is worth mentioning that the concentration values present in Table 1 describe the dynamic behavior of the decrease of the concentration of lead in the solution in contact with the hyacinth.

\section{Theoretical Framework}

As already mentioned, the goal of this work is the description of the dynamic behavior of lead sorption. In order to accomplish this task, the fractional kinetic model given by (1) a long with a correspondent initial condition is considered. This model resembles the classical one of first order used to describe processes with exponential relaxation; however, the differential operator is extended to a noninteger order, $\alpha$, with the aid of the fractional calculus generalization in order to cover a broad of situations which are not typically characterized by exponential processes. The choice of this model occurred because of the experimental data behavior the drop of concentration of lead in water resembles an exponential one, but with different asymptotic behavior:

$$
\frac{d^{\alpha} y(t)}{d t^{\alpha}}=-k \cdot y(t), \quad y(t=0)=y_{0} .
$$

Although there is a fractional order derivate, the model equation given by (1) is linear; therefore, the analytical solution can be obtained by Laplace transform technique [9]. By considering the Caputo approach [26] for the fractional derivate, (2) can be obtained after the Laplace transform inversion. This solution resembles the Mittag-Leffler function [27] with two parameters [28]:

$$
y(t)=y_{0} \cdot\left[\sum_{j=0}^{\infty} \frac{(-k)^{j} \cdot t^{\alpha \cdot(j+1)-1}}{\Gamma(\alpha \cdot(j+1))}\right] .
$$

For the sake of comparison, the classical first order model, given by (3) has also been taken into account. It is important to stress that (3) can be obtained by setting $\alpha$ equal to 1 in (2). This happens due to the generalization capability provided by the Mittag-Leffler based function present in the solution of (1):

$$
\frac{d y(t)}{d t}=-k \cdot y(t), \quad y(t=0)=y_{0} \longrightarrow y(t)=y_{0} \cdot e^{-k \cdot t} .
$$

Depending on the value of the order $\alpha$ of the derivative, the Mittag-Leffler function kernel present in (2) turns into a different mathematical function, as mentioned; if $\alpha=1$, an exponential function shows up. Therefore, one must pay attention that, as mentioned above, $\alpha$ is not an ordinary parameter, as it not only provides a better the data fitting but it also turns the sum of (2) into the best mathematical function to describe the experimental behavior.

The statistical validation methodology steps used were reported by Gomes et al. [10], where further details can be obtained. The parameter estimation task involved the determination of the parameters $\alpha$ and $k$ of the fractional model, (2), and the parameter $k$ of the integer order model, (3). This task considered an objective function given by (4). A genetic algorithm was used to avoid a local minimum value of the objective function:

$$
F_{\mathrm{OBJ}}=\sum_{p=1}^{\mathrm{NR}} \sum_{i=1}^{\mathrm{NE}}\left(y_{p, i}^{E}(t)-y_{p, i}^{M}(t)\right)^{2} .
$$


TABLE 2: Parameters and confidence interval for APPROACH01.

\begin{tabular}{|c|c|c|c|c|c|c|}
\hline & Model & Parameter & $\begin{array}{c}\text { Parameter standard } \\
\text { deviation } \delta_{\theta}\end{array}$ & $\begin{array}{l}\text { Parameter confidence interval } \\
\qquad \theta_{i} \pm z_{(95 \%)} \cdot \delta_{\theta_{i}}\end{array}$ & $r$ & $F_{\mathrm{OBJ}}$ \\
\hline \multirow{6}{*}{ RUN01 } & \multirow{2}{*}{ FRAC01 } & $k: 0.728$ & 0.239 & $0.73 \pm 0.47$ & \multirow{2}{*}{0.99} & \multirow{2}{*}{$3.64 \cdot 10^{-3}$} \\
\hline & & $\alpha: 0.203$ & 0.027 & $0.20 \pm 0.05$ & & \\
\hline & \multirow{2}{*}{ FRAC02 } & $k: 0.728$ & 0.221 & $0.73 \pm 0.43$ & \multirow[t]{2}{*}{0.99} & \multirow[t]{2}{*}{$3.64 \cdot 10^{-3}$} \\
\hline & & $\alpha: 0.203$ & 0.025 & $0.20 \pm 0.05$ & & \\
\hline & INT01 & $k: 4.775$ & 0.498 & $4.8 \pm 1.0$ & 0.98 & $1.91 \cdot 10^{-2}$ \\
\hline & INT02 & $k: 4.775$ & 0.438 & $4.8 \pm 0.8$ & 0.98 & $1.91 \cdot 10^{-2}$ \\
\hline
\end{tabular}

The experimental variance matrix, $V_{y^{E}}$, is given by (5), where the hypothesis of constant variance, $\delta_{\left(y^{E}\right)}^{2}$, was considered valid. Besides, experimental covariance was not taken into account:

$$
\begin{gathered}
{\left[V_{y^{E}}\right]_{(\mathrm{NE} \cdot \mathrm{NR} \times \mathrm{NE} \cdot \mathrm{NR})}=\delta_{\left(y^{E}\right)}^{2} \cdot\left[\begin{array}{cccc}
1 & 0 & \cdots & 0 \\
0 & 1 & \cdots & 0 \\
\vdots & \vdots & \ddots & \vdots \\
0 & 0 & \cdots & 1
\end{array}\right]} \\
\delta_{y^{E}}^{2}=\sum_{p=1}^{\mathrm{NR}} \sum_{i=1}^{\mathrm{NE}} \frac{\left(y_{p, i}^{E}(t)-y_{p, i}^{M}(t)\right)^{2}}{(\mathrm{NR} \cdot \mathrm{NE})-\mathrm{NP}} .
\end{gathered}
$$

In this work, the parametric variance analysis considered two different approaches, with the complete Hessian matrix $\left[H_{\theta}\right]$ and with a simplified Hessian matrix $[B]$, given by $(6)$ and $(7)$, respectively. The elements of the matrix $\left[H_{\theta}\right]$ are given by (8), while the elements of $[B]$ consider only the first order derivates of (8). In the following expressions, $\theta$ refers to the parameter set $\{\alpha ; k\}$ to be estimated. Further details can be found in the literature [10]:

$$
\begin{gathered}
{\left[V_{\theta}\right]_{(\mathrm{NP} \times \mathrm{NP})}} \\
=\left[H_{\theta}^{-1}\right]_{(\mathrm{NP} \times \mathrm{NP})} \\
\cdot\left(\left[G_{y}\right]_{(\mathrm{NP} \times \mathrm{NE} \cdot \mathrm{NR})} \cdot\left[V_{y^{E}}\right]_{(\mathrm{NE} \cdot \mathrm{NR} \times \mathrm{NE} \cdot \mathrm{NR})}\right. \\
\left.\cdot\left[G_{y}^{T}\right]_{(\mathrm{NE} \cdot \mathrm{NR} \times \mathrm{NP})}\right) \cdot\left[H_{\theta}^{-1}\right]_{(\mathrm{NP} \times \mathrm{NP})}, \\
{\left[V_{\theta}\right]_{(\mathrm{NP} \times \mathrm{NP})}} \\
=\left(\left[B^{T}\right]_{(\mathrm{NP} \times \mathrm{NE} \cdot \mathrm{NR})} \cdot\left[V_{y^{E}}^{-1}\right]_{(\mathrm{NE} \cdot \mathrm{NR} \times \mathrm{NE} \cdot \mathrm{NR})}\right. \\
\left.\cdot[B]_{(\mathrm{NE} \cdot \mathrm{NR} \times \mathrm{NP})}\right)^{-1}, \\
\frac{\partial^{2} F_{\mathrm{OBJ}}}{\partial \theta_{i} \partial \theta_{J}}=2 \cdot\left(\frac{\partial y^{M}}{\partial \theta_{i}}\right)^{T} \cdot V_{y^{E}}^{-1} \\
\cdot\left(\frac{\partial y^{M}}{\partial \theta_{j}}\right)-2 \cdot\left(\frac{\partial^{2} y^{M}}{\partial \theta_{i} \partial \theta_{j}}\right)^{T} \cdot V_{y}^{-1} \cdot\left(y^{E}-y^{M}\right) .
\end{gathered}
$$

Finally, joint parameter confidence regions were generated. As reported by Gomes et al. [10], (9) refers to joint confidence regions obtained by the linearization of the objective function, which are typically of ellipsoidal shape. On the other hand, (10) presents the formula used to obtain joint confidence region considering the nonlinear features of the objective function. This region is not necessarily ellipsoidal and it is usually much larger than the one obtained by (9). In these expressions, $\theta$ is the set of parameters, $\theta^{*}$ is the set of estimated parameters, $F_{\mathrm{NP},(\mathrm{NE}-\mathrm{NP})}^{(95 \%)}$ is the value of the Fischer distribution with $95 \%$ of confidence and NP and (NE - NP) are degrees of freedom, NE is the number of experimental points of a given experimental run, and NR is the number of experimental runs:

$$
\begin{gathered}
{\left[\left(\theta-\theta^{*}\right)^{T}\right]_{(1 \times \mathrm{NP})} \cdot\left[V_{\theta}^{-1}\right]_{(\mathrm{NP} \times \mathrm{NP})} \cdot\left[\left(\theta-\theta^{*}\right)\right]_{(\mathrm{NP} \times 1)}} \\
\leq F_{\mathrm{OBJ}}\left(\theta^{*}\right) \cdot \frac{\mathrm{NP}}{\mathrm{NE}-\mathrm{NP}} \cdot F_{\mathrm{NP},(\mathrm{NE}-\mathrm{NP})}^{(95 \%)} \\
F_{\mathrm{OBJ}}(\theta) \leq F_{\mathrm{OBJ}}\left(\theta^{*}\right) \cdot\left(1+\frac{\mathrm{NP}}{\mathrm{NE}-\mathrm{NP}} \cdot F_{\mathrm{NP},(\mathrm{NE}-\mathrm{NP})}^{(95 \%)}\right) .
\end{gathered}
$$

The modeling work was carried out considering two different approaches. In the first one, named APPROACH01, the experimental set RUN01 was used for parameter estimation of each model, that is, (2) and (3), and the resulting equations were used to validate the behavior exhibited by the experimental data set RUN 02. In the second one, APPROACH02, both experimental sets were used to estimate a global set of parameters for each model. It must be mentioned that, for each of these approaches, the parametric variance was calculated using the two different approaches mentioned in the last paragraph, that is, (6) and (7).

\section{Results and Discussions}

4.1. APPROACH01 Results. Table 2 presents a summary of the results of the parameter estimation. FRAC01 refers to the parametric analysis of the fractional model, (2), using the complete hessian matrix (6) to evaluate the variance of the estimated parameters. FRAC02 refers to the parametric analysis of the fractional model, (2), using the simplified hessian matrix (7) to evaluate the variance of the estimated parameters. INT01 refers to the parametric analysis of the integer order model, (3), using the complete hessian matrix (6) to evaluate the variance of the estimated parameters. 


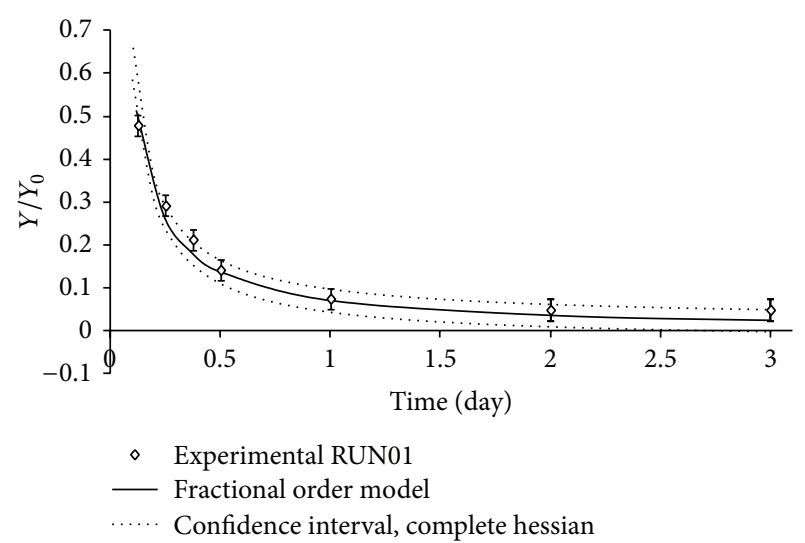

FIGURE 1: Experimental and predicted values against time Experiment 01, fractional order model, APPROACH01.

INT02 refers to the parametric analysis of the integer order model, (3), using the simplified hessian matrix (7) to evaluate the variance of the estimated parameters. From the results reported in Table 1, it is possible to observe that the choice of calculation of parametric variance has a little influence on the parameter deviation. This probably happened because as the data fit was good, the term $\left(y^{E}-y^{M}\right)$, corresponding to the difference between experimental results and model predictions, has a small value.

The objective function $\left(F_{\mathrm{OBJ}}\right)$ has a low value for fractional model when compared to the integer order model; however, for the fractional model, the value is one order of magnitude lower. This probably happened because the fractional model could better describe the memory effects $[10,21]$ present in the sorption process. Also, one can note that the parameter $\alpha$, when estimated, also shapes the sum to a specific mathematical function, providing a better fit than the integer order model. It is also important to stress that due to its intrinsic features, the fractional order model also considers memory effects, which happens due to the convolution integral of the fractional derivate approach considered. This type of effect is constantly present in irregular, disordered, and fractal media diffusion and mass transfer [29]. Taking the memory effects into account significantly improves the modeling results, such as in systems like the irregular shape of the hyacinth leaves present in the interface with the lead aqueous solution.

It is worth mentioning that the correlation coefficient $(r)$ was very good in both cases, presenting values equal to 0.99 . Moreover, the estimated values of parameters $k$ and $\alpha$ are greater than their respective parametric deviation for all models; consequently these parameters can be regarded as statistically significant.

Figures 1 and 2 show a comparison between experimental data and model predictions plotted against time for model FRAC01 and INT01, where parameter variance was obtained using the complete Hessian matrix. Again, the fractional model described the experimental data more adequately than the integer order model, as expected after analyzing Table 1.

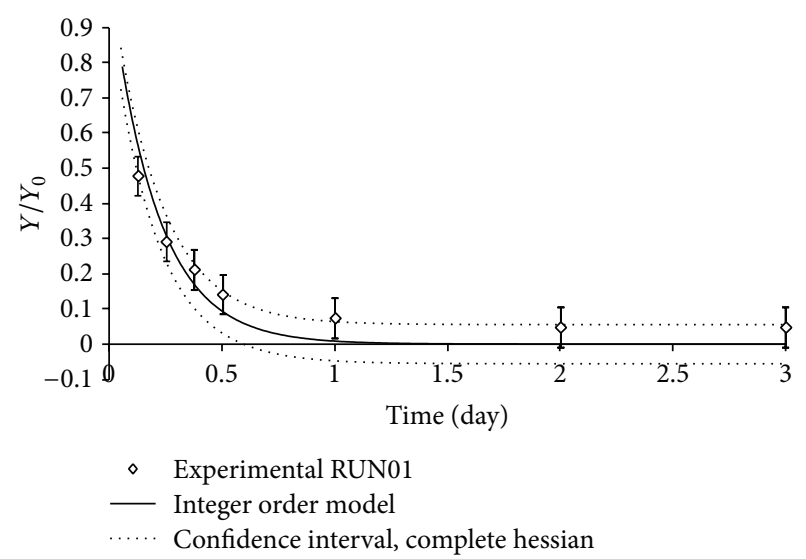

FIGURE 2: Experimental and predicted values against time Experiment 01, integer order model, APPROACH01.

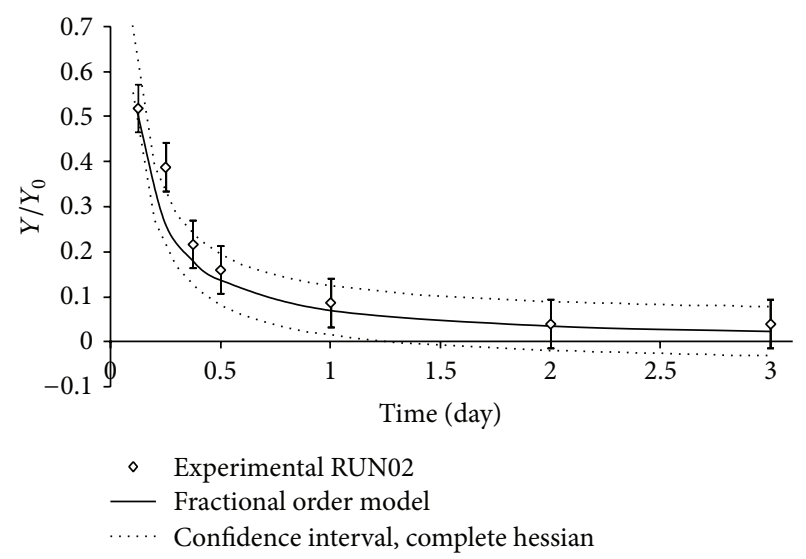

FIgURE 3: Experimental and predicted values against time Experiment 02, fractional order model, APPROACH01.

In these figures, one can see the confidence interval of the model predictions. It can be observed that for both models, the experimental data lie within these regions, but in the fractional model analysis the obtained regions are smaller showing that the model predictions tend to be more accurate.

Figures 3 and 4 present the prediction/validation of experimental set RUN02 using the model parameters estimated using only experimental RUN01. It can be clearly seen that the model predictions of the fractional model are better than the predictions of the integer order model, as the experimental points are closer to the model prediction and the confidence region is smaller. Finally, it is worth mentioning that this result shows the extrapolation capability of operating conditions as the amount of lead in experiment RUN02 is 50\% higher than in experiment RUN01.

Figure 5 shows the joint parameter confidence (ellipsoid) region, considering a confidence level of $95 \%$ and the linearization of the estimation problem as mentioned before (see (9)). Figure 6 shows the joint parameter confidence region obtained considering the nonlinear feature of the estimation problem; see (10). In both cases, it can be seen that parameter $\alpha$ does not reach a value equal to 1 , proving that the fractional 
TABLE 3: Parameters and confidence interval for APPROACH02.

\begin{tabular}{|c|c|c|c|c|c|c|}
\hline & Model & Parameter & $\begin{array}{l}\text { Parameter standard } \\
\text { deviation } \delta_{\theta}\end{array}$ & $\begin{array}{l}\text { Parameter confidence interval } \\
\qquad \theta_{i} \pm z_{(95 \%)} \cdot \delta_{\theta_{i}}\end{array}$ & $r$ & $F_{\mathrm{OBJ}}$ \\
\hline \multirow{6}{*}{ RUN01 + RUN02 } & \multirow{2}{*}{ FRAC01 } & $k: 0.650$ & 0.231 & $0.65 \pm 0.45$ & \multirow[t]{2}{*}{0.99} & \multirow[t]{2}{*}{$1.78 \cdot 10^{-2}$} \\
\hline & & $\alpha: 0.201$ & 0.029 & $0.20 \pm 0.05$ & & \\
\hline & \multirow{2}{*}{ FRAC02 } & $k: 0.650$ & 0.226 & $0.65 \pm 0.44$ & \multirow[t]{2}{*}{0.99} & \multirow[t]{2}{*}{$1.78 \cdot 10^{-2}$} \\
\hline & & $\alpha: 0.201$ & 0.028 & $0.20 \pm 0.05$ & & \\
\hline & INT01 & $k: 4.412$ & 0.256 & $4.4 \pm 1.0$ & 0.99 & $4.00 \cdot 10^{-2}$ \\
\hline & INT02 & $k: 4.412$ & 0.201 & $4.4 \pm 0.8$ & 0.99 & $4.00 \cdot 10^{-2}$ \\
\hline
\end{tabular}

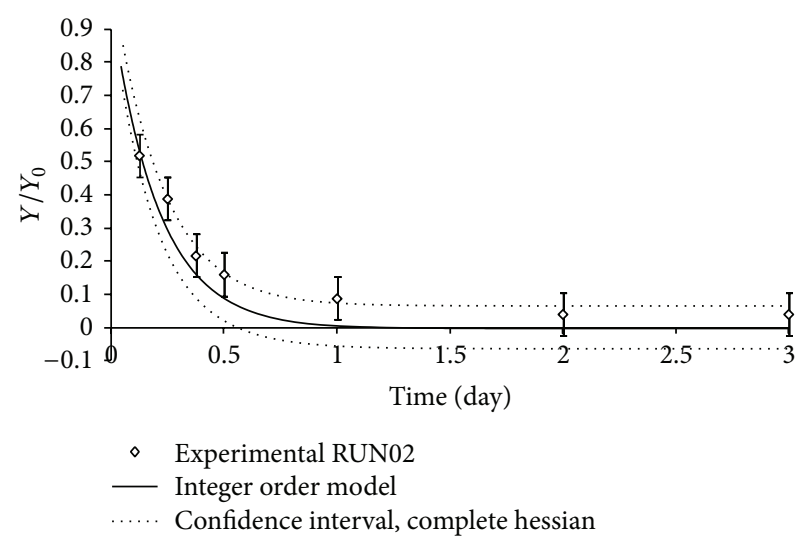

FIGURE 4: Experimental and predicted values against time Experiment 02, integer order model, APPROACH01.

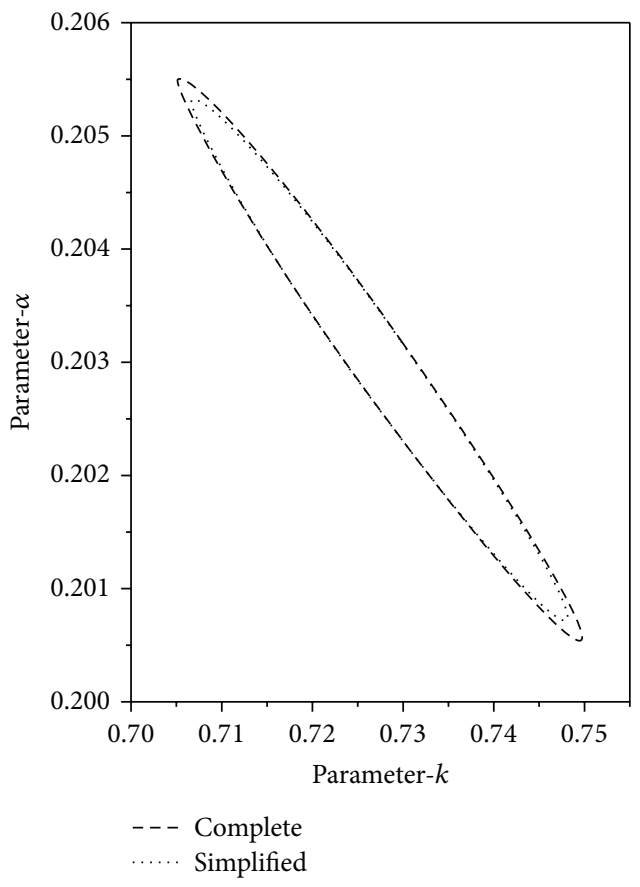

FIGURE 5: Confidence region (ellipsoid) for simplified and complete Hessian matrix, APPROACH01.

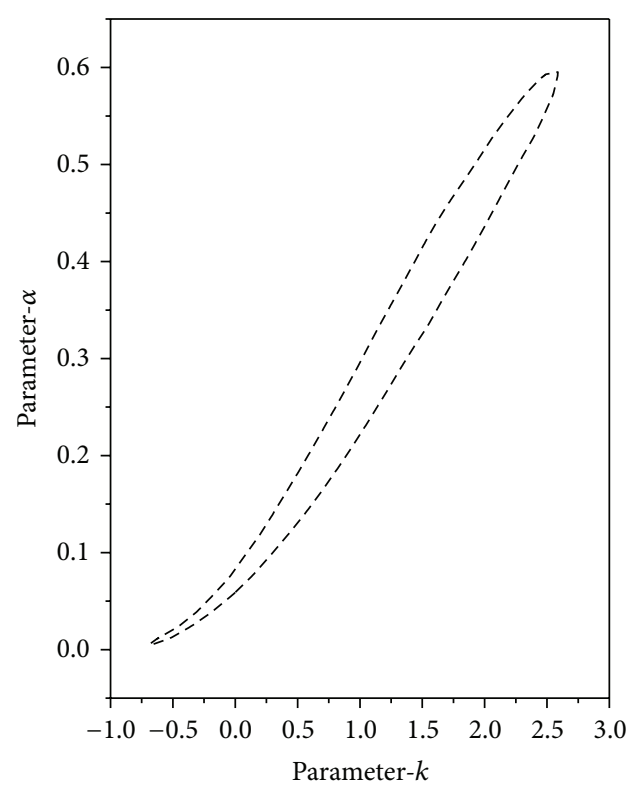

FIGURE 6: Confidence region (nonlinear), APPROACH01.

order estimated is statistically not integer, allowing one to conclude that the model is in fact fractional.

4.2. APPROACHO2 Results. Table 3 presents a summary of the results of the parameter estimation. FRAC01 refers to calculation using complete hessian matrix (6) of the fractional model and FRAC02 refers to the simplified hessian matrix (7). The same observation is valid for the integer order model (INT01 and INT02). From this estimation task result, it is possible to observe that the choice of calculation of parametric variance has practically no influence on the parameter deviation for this case. This probably happened because, as both sets were simultaneously used, the larger number of experimental points led to a lower experimental variance (5).

The objective function $\left(F_{\mathrm{OBJ}}\right)$ has a lower value for fractional model when compared to the integer order model. It is worth mentioning that the correlation coefficient $(r)$ was very good in both cases, presenting values over 0.99 . Besides, the parameters $k, e$, and $\alpha$ were greater than their 


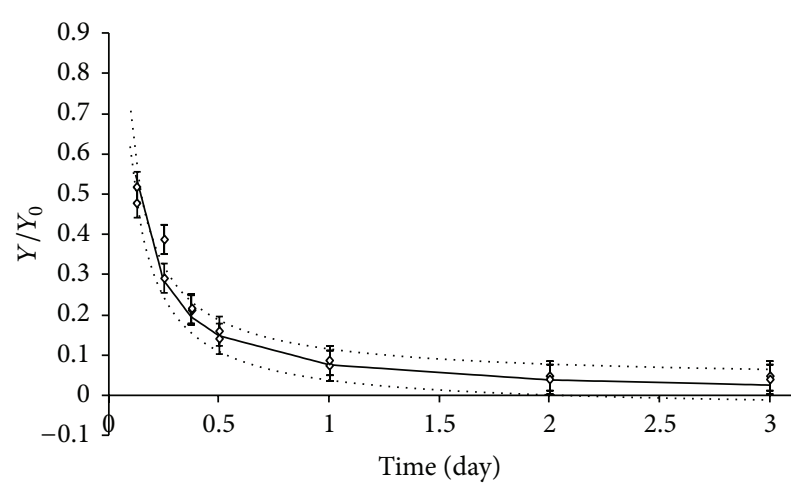

- Experimental: RUN01 + RUN02

- Fractional order model

..... Confidence interval, complete hessian

FIGURE 7: Experimental and predicted values against time Experiments 01 and 02, fractional order model, APPROACH02.

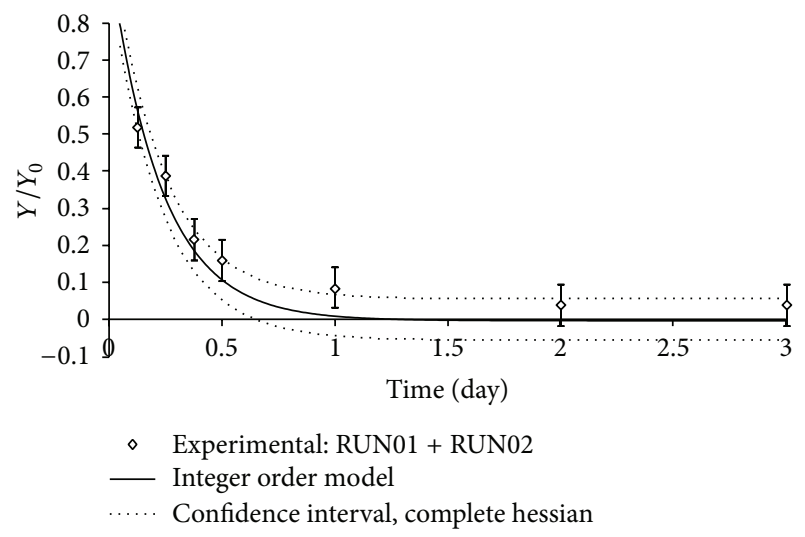

FIGURE 8: Experimental and predicted values against time Experiments 01 and 02, INT model, APPROACH02.

respective parametric deviation in all models; consequently the parameters are statistically significant.

Figure 7 shows a comparison of experimental data and model predictions plotted against the independent variable for fractional order model. Figure 8 presents the same plot but with considering the integer order model. The deviation was smaller than in previous approach. This is probably because both datasets were used in the estimation problem. However, in both cases it can be observed that the integer order model prediction was slightly worse and has more deviations.

Figure 9 presents the confidence region. As can be seen, independently of the Hessian matrix considered, that is, complete or simplified, the two regions are very close. This happens because the term containing the second order derivate in (8) is closer to zero due to the better fit in APPROACH02 as both datasets were simultaneously used. Finally, Figure 10 shows the joint confidence region considering the nonlinear features of the problem. Again, this region is larger than the one obtained for the linear problem and it also shows that the order of the derivative is smaller than 1 , indicating a fractional process.

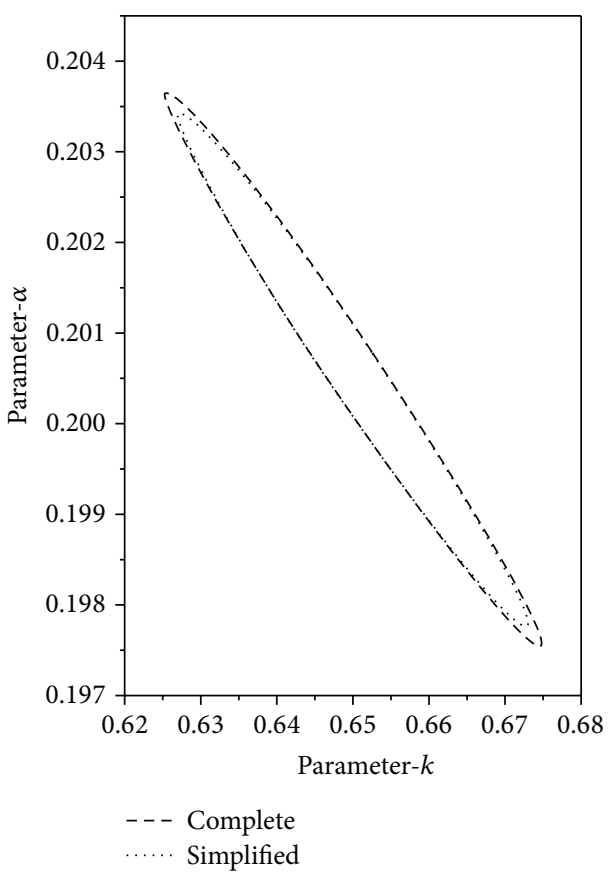

FIGURE 9: Confidence region with simplified and complete Hessian matrix, APPROACH02.

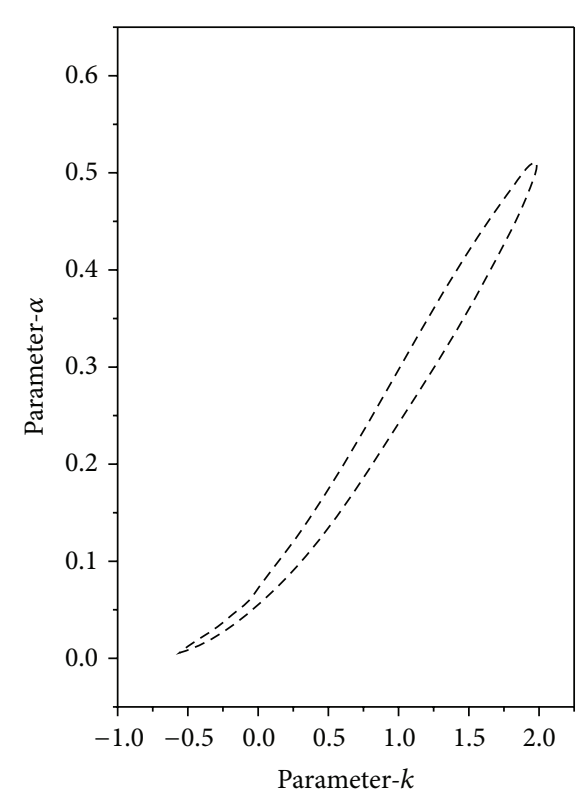

Figure 10: Confidence region (nonlinear), APPROACH02.

\section{Conclusion}

The main purpose of this work was to present a model to describe lead sorption kinetics using water hyacinths taking into account fractional calculus issues and to carry out an error analysis to validate fractional nature of the model. The parameter estimation tasks were made by two different approaches, one of them considering one experimental 
dataset and the other one for validation, and the second approach with all experimental datasets.

Parametric variance matrix was calculated in two different ways, but for this problem the results were roughly the same. From parametric variance, the confidence regions could be obtained considering a linearization of the problem or the nonlinear problem itself.

It was concluded that the fractional order model presented better results than the integer order model. Besides, the noninteger order model was in fact fractional, as statistically proven by the error analysis and parameter confidence region. The model in APPROACH01 was satisfactorily used for extrapolation of experimental conditions, condition not reached by integer order model. In this way, the developed model can be applied with success in the heavy metal sorption modeling.

\section{Nomenclature}

$\begin{array}{ll}F_{\mathrm{OBJ}}: & \text { Objective function } \\ F_{\mathrm{NP},(\mathrm{NE}-\mathrm{NP})}^{(95 \%)} & \begin{array}{l}\text { Fischer distribution with } 95 \% \text { of } \\ \text { confidence and NP and }(\mathrm{NE}-\mathrm{NP}) \text { degrees } \\ \end{array} \\ i, j, p: & \begin{array}{l}\text { of freedom } \\ \text { Counter }\end{array} \\ k: & \text { Kinetic sorption constant }\left(\text { day }^{-1}\right) \\ \mathrm{NE}: & \text { Number of experimental points of a given } \\ & \text { experimental run } \\ \mathrm{NP}: & \text { Number of estimated parameters } \\ \mathrm{NR}: & \text { Number of experimental runs } \\ t: & \text { Time (day) } \\ V: & \text { Variance matrix } \\ y: & \text { Lead concentration (mg/L) } \\ y_{0}: & \text { Initial lead concentration (mg/L) } \\ z_{95 \%}: & \text { Normal distribution value obtained with } \\ & \text { confidence level of } 95 \% .\end{array}$

\section{Superscript}

E: Experimental

M: Model.

\section{Greek Symbols}

$\alpha$ : Fractional order derivative

$\theta:$ Parameter $\{\alpha$ or $k\}$

$\theta^{*}:$ Estimated parameter $\{\alpha$ or $k\}$

$\Gamma$ : Gamma function

$\delta^{2}$ : Variance.

\section{Conflict of Interests}

The authors declare that there is no conflict of interests regarding the publication of this paper.

\section{Acknowledgments}

The authors are gratefully thankful for CNPQ and CAPES.

\section{References}

[1] P. Gottesfeld, G. Kuepouo, S. Tetsopgang, and K. Durand, "Lead concentrations and labeling of new paint in cameroon," Journal of Occupational and Environmental Hygiene, vol. 10, no. 5, pp. 243-249, 2013.

[2] H. J. Mansoorian, A. H. Mahvi, and A. J. Jafari, "Removal of lead and zinc from battery industry wastewater using electrocoagulation process: influence of direct and alternating current by using iron and stainless steel rod electrodes," Separation and Purification Technology, vol. 135, pp. 165-175, 2014.

[3] M. Ekinci, E. Ceylan, H. H. Cagatay et al., "Occupational exposure to lead decreases macular, choroidal, and retinal nerve fiber layer thickness in industrial battery workers," Current Eye Research, vol. 39, no. 8, pp. 853-858, 2014.

[4] F. H. Were, M. C. Moturi, P. Gottesfeld, G. A. Wafula, G. N. Kamau, and P. M. Shiundu, "Lead exposure and blood pressure among workers in diverse industrial plants in Kenya," Journal of Occupational and Environmental Hygiene, vol. 11, no. 11, pp. 706-715, 2014.

[5] H. Sis and T. Uysal, "Removal of heavy metal ions from aqueous medium using Kuluncak (Malatya) vermiculites and effect of precipitation on removal," Applied Clay Science, vol. 95, pp. 1-8, 2014.

[6] Q. Wang, D. Zhang, S. Tian, and P. Ning, "Simultaneous adsorptive removal of methylene blue and copper ions from aqueous solution by ferrocene-modified cation exchange resin," Journal of Applied Polymer Science, vol. 131, no. 21, Article ID 41029, 2014.

[7] J. He and J. P. Chen, "A comprehensive review on biosorption of heavy metals by algal biomass: materials, performances, chemistry, and modeling simulation tools," Bioresource Technology, vol. 160, pp. 67-78, 2014.

[8] M. C. dos Santos and E. Lenzi, "The use of aquatic macrophytes (Eichhornia crassipes) as a biological filter in the treatment of lead contaminated effluents," Environmental Technology, vol. 21, no. 6, pp. 615-622, 2000.

[9] A. F. B. Duarte, J. M. G. Pereira, M. K. Lenzi, G. Gonçalves, R. Rossato, and E. K. Lenzi, "Diffusion equations and different spatial fractional derivatives," Acta Scientiarum-Technology, vol. 36, no. 4, pp. 657-662, 2014.

[10] E. M. Gomes, R. R. Araújo, M. K. Lenzi, F. R. Silva, and E. K. Lenzi, "Parametric analysis of a heavy metal sorption isotherm based on fractional calculus," Mathematical Problems in Engineering, vol. 2013, Article ID 642101, 10 pages, 2013.

[11] I. Langmuir, "The constitution and fundamental properties of solids and liquids. Part I. Solids," The Journal of the American Chemical Society, vol. 38, no. 2, pp. 2221-2295, 1916.

[12] H. M. F. Freundlich, "Über die Adsorption in Lösungen," Zeitschrift für Physikalische Chemie, vol. 57, no. A, pp. 385-470, 1906.

[13] O. Redlich and D. L. Peterson, "A useful adsorption isotherm," The Journal of Physical Chemistry, vol. 63, no. 6, p. 1024, 1959.

[14] D. D. Do, Adsorption Analysis: Equilibria and Kinetics, Imperial College Press, London, UK, 1st edition, 1998.

[15] S. Lagergren, "Kungliga Svenska Vetenskapsakademiens," Handlingar, vol. 24, pp. 1-39, 1898.

[16] Y. S. Ho, "Review of second-order models for adsorption systems," Journal of Hazardous Materials, vol. 136, no. 3, pp. 681689, 2006. 
[17] J. A. T. Machado, A. M. S. F. Galhano, M. S. F. Alexandra, and J. J. Trujillo, "On development of fractional calculus during the last fifty years," Scientometrics, vol. 98, no. 1, pp. 577-582, 2014.

[18] J. Hristov, "Redistribution of mass from a thin interlayer between two thick dissimilar media 1-D diffusion problem with a non-local condition," Thermal Science, vol. 17, no. 3, pp. 651664, 2013.

[19] E. Gonzalez, L. Dorčák, C. Monje, J. Valsa, F. Caluyo, and I. Petráš, "Conceptual design of a selectable fractional-order differentiator for industrial applications," Fractional Calculus and Applied Analysis, vol. 17, no. 3, pp. 697-716, 2014.

[20] R. Kopelman, "Fractal reaction-kinetics," Science, vol. 241, no. 4873, pp. 1620-1626, 1988.

[21] R. P. Meilanov, D. A. Sveshnikova, and O. M. Shabanov, "Fractal nature of sorption kinetics," The Journal of Physical Chemistry A, vol. 106, no. 48, pp. 11771-11774, 2002.

[22] F. Brouers and O. Sotolongo-Costa, "Generalized fractal kinetics in complex systems (application to biophysics and biotechnology)," Physica A: Statistical Mechanics and Its Applications, vol. 368, no. 1, pp. 165-175, 2006.

[23] S. Gaspard, S. Altenor, N. Passe-Coutrin, A. Ouensanga, and F. Brouers, "Parameters from a new kinetic equation to evaluate activated carbons efficiency for water treatment," Water Research, vol. 40, no. 18, pp. 3467-3477, 2006.

[24] M. C. Ncibi, B. Mahjoub, M. Seffen, F. Brouers, and S. Gaspard, "Sorption dynamic investigation of chromium(VI) onto Posidonia oceanica fibres: kinetic modelling using new generalized fractal equation," Biochemical Engineering Journal, vol. 46, no. 2, pp. 141-146, 2009.

[25] A. M. B. Hamissa, F. Brouers, M. C. Ncibi, and M. Seffen, "Kinetic modeling study on methylene blue sorption onto Agave americana fibers: fractal kinetics and regeneration studies," Separation Science and Technology, vol. 48, no. 18, pp. 28342842, 2013.

[26] M. Caputo, "Linear models of dissipation whose $q$ is almost frequency independent-II," Geophysical Journal International, vol. 13, no. 5, pp. 529-539, 1967.

[27] G. M. Mittag-Leffler, "Sur la nouvelle function E(x)," Comptes Rendus de l'Académie des Sciences, vol. 137, pp. 554-558, 1903.

[28] I. Podlubny, Fracitonal Differential Equations, Academic Press, New York, NY, USA, 1st edition, 1999.

[29] M. Giona and H. E. Roman, "A theory of transport phenomena in disordered systems," The Chemical Engineering Journal, vol. 49, no. 1, pp. 1-10, 1992. 


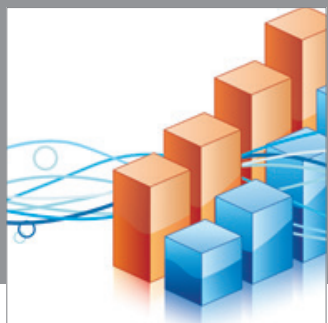

Advances in

Operations Research

mansans

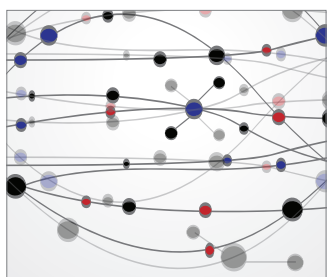

The Scientific World Journal
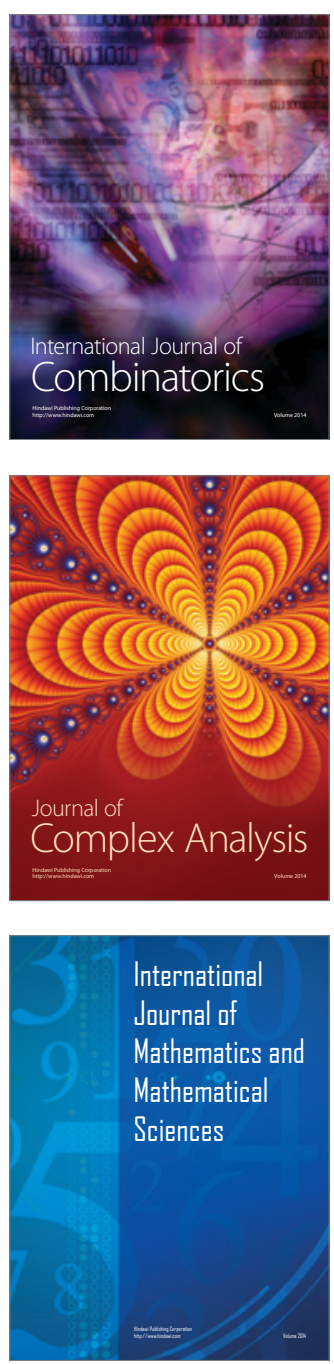
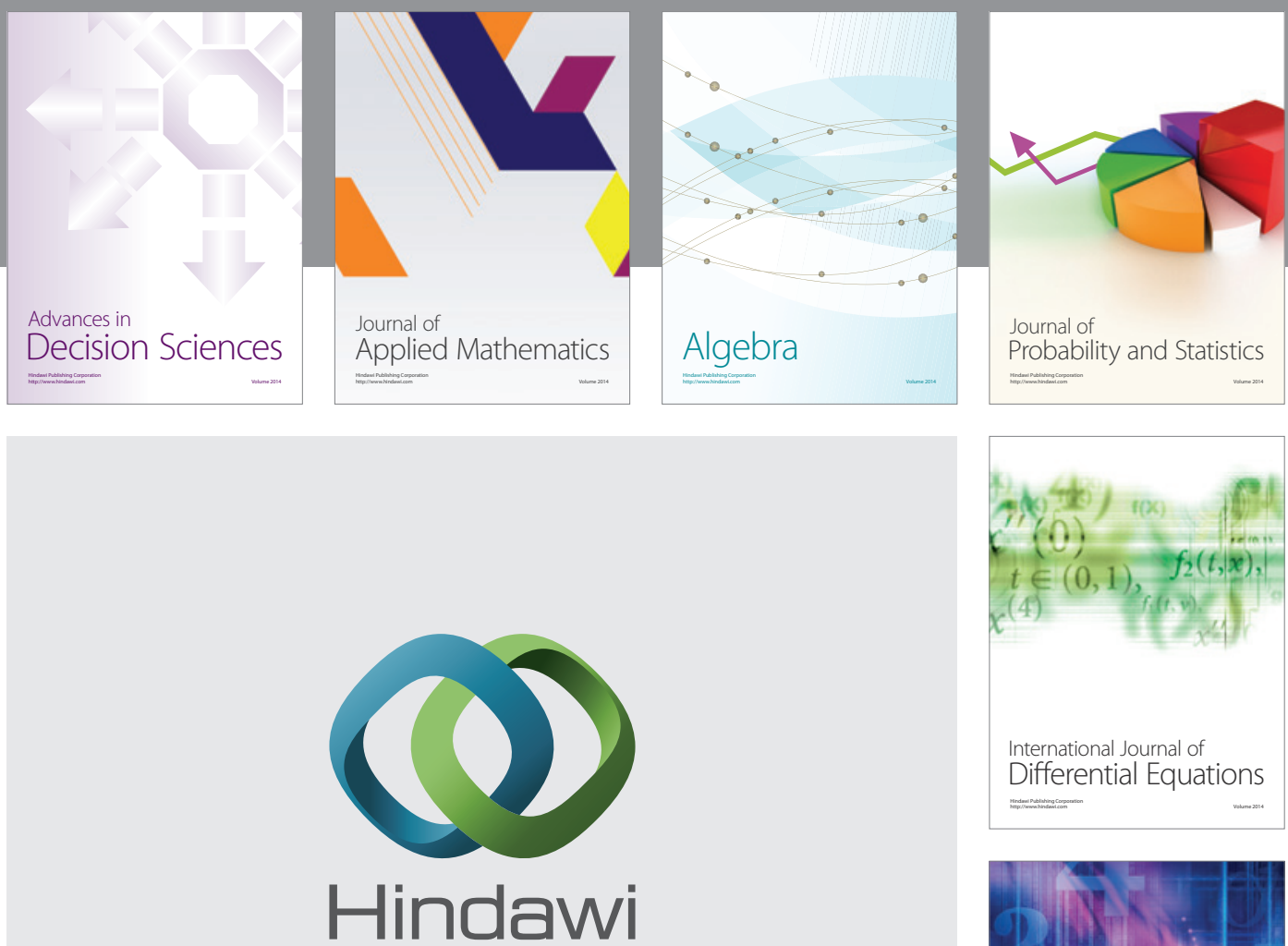

Submit your manuscripts at http://www.hindawi.com
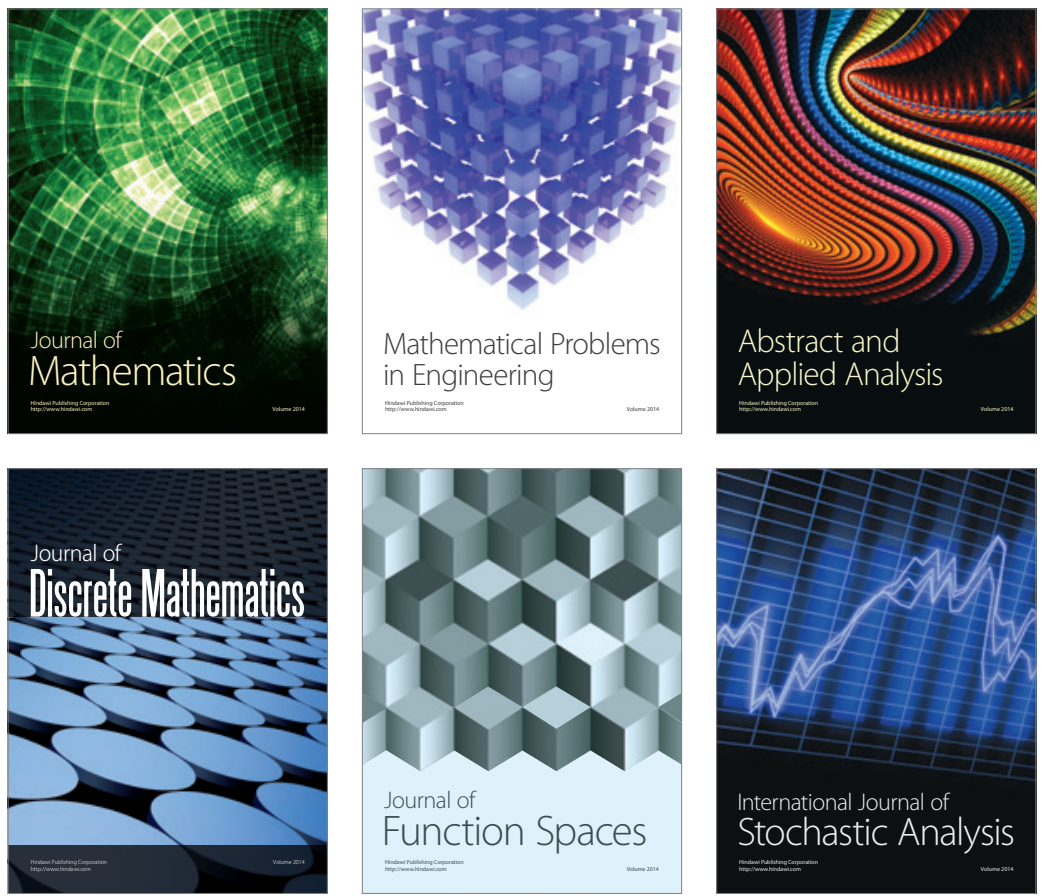

Journal of

Function Spaces

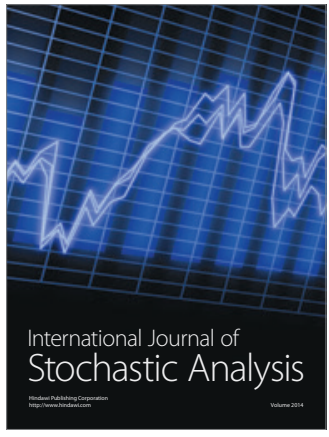

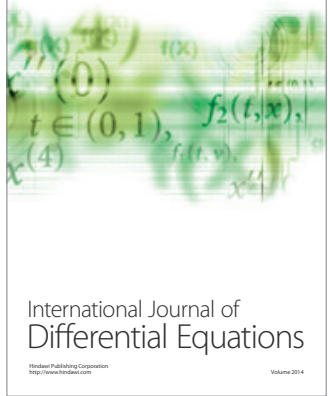
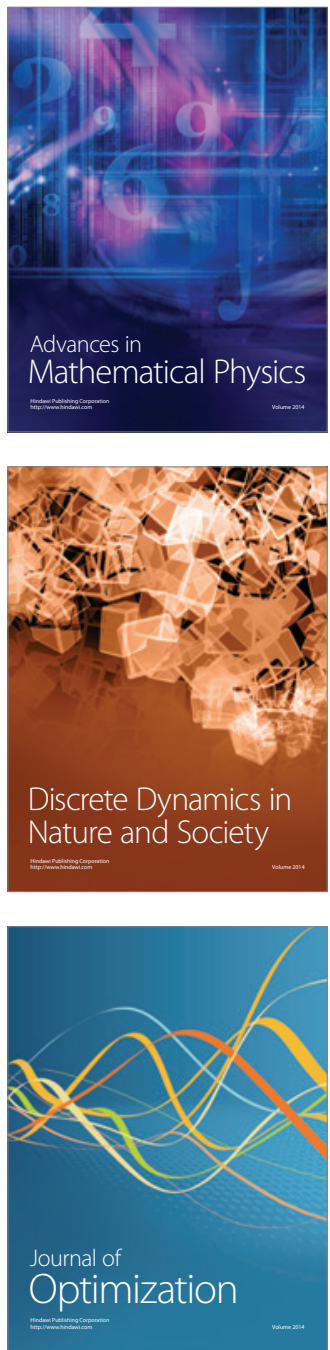